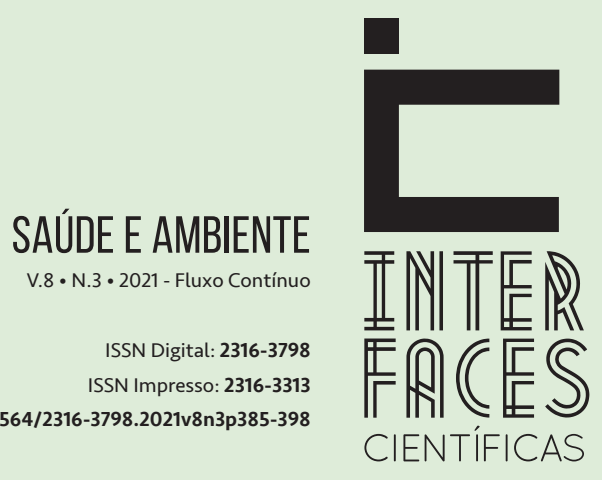

\section{AVALIAÇÃO DA COMPOSIÇ̃̃O DE NUTRICOSMÉTICOS UTILIZADOS NO TRATAMENTO DE CABELO}

EVALUATION OF THE COMPOSITION OF NUTRICOSMETICS USED IN HAIR TREATMENT

\section{EVALUACIÓN DE LA COMPOSICIÓN DE NUTRICOSMÉTICOS UTILIZADOS EN EL TRATAMIENTODE CABELLO}

Nathalia Caroline Mandarino Silva Ingrid Gabriele Silva Maciel $^{2}$

Eliane Freire de Oliveira Fernandes ${ }^{3}$ Heriberto Alves dos Anjos

\section{RESUMO}

Os padrões de beleza têm aumentado a demanda por suplementos nutricionais, denominados nutricosméticos, que apresentam diversas finalidades estéticas, dentre elas, benefícios no tratamento e saúde do cabelo. 0 presente estudo tem por finalidade realizar uma análise da composição nutricional desses produtos encontrados no mercado. Para isso, foi realizada uma pesquisa exploratória e descritiva dos suplementos para cabelos achados em farmácias e lojas de produtos naturais no município de Aracaju/SE, com a finalidade de obter dados presentes nos rótulos quanto a composição nutricional, indicação de uso, posologia, nome e marca. Dessa forma, foram encontrados e tabulados 75 produtos de 73 marcas diferentes, em que 22 deles são específicos para o cabelo, 29 para cabelos e unhas e 24 para cabelos, unhas e pele, que atendessem as características do estudo. Com relação à composição nutricional foram encontrados como destaque: biotina em $88 \%$ dos produtos; piridoxina em $82 \%$; o zinco em $80 \%$; o selênio em $61 \%$; as vitaminas $\mathrm{C}$ e $\mathrm{E}$ em $60 \%$ dos produtos analisados. Além dos minerais e vitaminas encontrados nos nutricosméticos, identificaram-se também 29 compostos diferentes; no entanto, eles aparecem em poucos suplementos. Observou-se que a literatura atribui aos nutrientes encontrados um papel no crescimento e fortalecimento do cabelo. Entretanto, estudos que mostram a efetividade dessa função ainda são escassos; logo, são necessárias mais pesquisas sobre o tema, além de orientação profissional na prescrição de nutricosméticos em casos de carência para adequar o padrão alimentar com a necessidade de suplementação nutricional. 


\section{PALAVRAS-CHAVE}

Cabelo. Estética. Nutrientes.

\section{ABSTRACT}

Beauty standards have increased the demand for nutritional supplements, named nutricosmetics, which serve several aesthetic purposes, including benefits for hair treatment. The present study aims to analyze the nutritional composition of some of these products found on the market. To do so, an exploratory and descriptive research of hair supplements localized in pharmacies and natural products stores in the city of Aracaju-SE was carried out; the goal was to obtain, from the labels, data on nutritional composition, indication of use and dosage, name and brand. Consequently, 75 products of 73 different brands met the characteristics of the study and were tabulated: 22 of them are for hair, 29 are for hair and nails, 24 are for hair, nails and skin. With regard to nutritional composition, the following components were highlighted: biotin was in $88 \%$ of the products; pyridoxine in $82 \%$; zinc in $80 \%$; selenium present in $61 \%$; vitamins $C$ and $E$ in $60 \%$ of the analyzed products. In addition to the minerals and vitamins found in the products, the presence of 29 compounds was identified; however, they appeared in a few supplements. The literature stablishes a relationship between these nutrients and the growth and strengthening of hair. Nonetheless, studies showing the effectiveness of such association are still scarce; therefore, more research in this area is essential. Additionally, the guidance of a professional is required for the prescription of these products in case of nutritional deficiency, so the dietary pattern can be adapted to the need for nutritional supplementation.

\section{KEYWORDS}

Hair. Esthetics. Nutrients.

\section{RESUMEN}

Los padrones de belleza han aumentado la demanda por suplementos nutricionales, llamados nutricosméticos, que presentan distintas finalidades estéticas, entra ellas, beneficios en el tratamiento y salud del pelo. El presente estudio tiene por finalidad realizar un análisis de la composición nutricional de esos productos encontrados en el mercado. Para eso, fue realizada una pesquisa exploratoria y descriptiva de los productos para pelos, encontrados en farmacias y tiendas de productos biológicos 
en la ciudad de Aracaju (Sergipe), con la finalidad de obtener datos presentes en las etiquetas acerca de la composición nutricional, indicación de uso, posología, nombre y marca. De esa forma, fueron encontrados y tabulados 75 productos de 73 marcas distintas, de los cuales, 22 son específicos para el pelo, 29 para pelos y uñas y 24 para pelos, uñas y piel, y que atendiesen a las características del estudio. Con relación a la composición nutricional fueran encontrados como destaque: biotina en $88 \%$ de los productos; piridoxina en $82 \%$; el zinco en $80 \%$; el selenio en $61 \%$; las vitaminas C y E en $60 \%$ de los productos analizados. Además de los minerales y vitaminas encontrados en los nutricosméticos, se identificó también 29 compuestos distintos, pero ellos aparecen en pocos suplementos. Se observó que la literatura atribuye a los nutrientes encontrados un papel en el crecimiento y fortalecimiento del pelo. Sin embargo, estudios que muestran la efectividad de esa función aún son pocos; así, son necesarias más pesquisas acerca de la temática, además de la orientación profesional en la prescripción de nutricosméticos en casos de carencia para adecuar el padrón alimentar con la necesidad de suplementación nutricional.

\section{PALABRAS CLAVE}

Cabello, Estética, Nutrientes.

\section{INTRODUÇ̄̃̃O}

O culto ao corpo e a beleza tem aumentado a demanda do mercado quanto aos produtos provenientes da indústria de cosméticos. De acordo com Mota (2008), a indústria, por intermédio dos meios de comunicação, atribui-se a criar desejos e ressaltar imagens de padrões estéticos e de beleza. Diante desse cenário, a indústria de cosméticos tem bastante relevância em virtude do constante investimento em inovação da indústria e da tecnologia de ponta na formulação desses produtos (PUJOL, 2011).

Especialistas da indústria cosmética, visando suprir os desejos do mercado, associados à indústria de alimentos, analisam diversas fusões de bioativos e nutrientes que auxiliem na busca da beleza a fim de responder às demandas dos consumidores que buscam hábitos mais saudáveis e resultados estéticos. Dessa forma, surgiram os nutricosméticos, que tem como intenção atender as necessidades nutricionais do organismo "de dentro para fora" com a finalidade estética (ALFAWAZ et al., 2017; CÂNDIDO et al., 2020).

Os nutricosméticos se apresentam em diversas formas farmacêuticas, dentre elas: pastilhas, cápsulas, comprimidos, líquidos, dentre outros e seu uso é feito por meio da via oral, o que assegura um maior aporte de vitaminas, aminoácidos, compostos bioativos e proteínas em proporções compatíveis para satisfazer os benefícios almejados. Os fatores determinantes na busca por esses produtos pelos consumidores são ações antioxidante, antienvelhecimento, antiqueda capilar, hidratação, fortificação capilar e dérmica, fotoproteção e redução da adiposidade localizada (GONÇALVES, 2016). 
Uma alimentação balanceada é indispensável para a saúde de todos, visto que uma ingestão alimentar inadequada pode levar a uma deficiência nutricional e o consumo de alimentos em quantidades calóricas acima do necessário para o indivíduo, pode acarretar problemas de saúde futuros, como as doenças cardiovasculares (ALFAWAZ et al., 2020; WITT; SCHNEIDER, 2011).

É de grande relevância científica estudar a composição nutricional dos suplementos alimentares para cabelos e unhas, devido à crescente oferta e demanda desses produtos no mercado, também em virtude do escasso número de trabalhos publicados nessa temática (ALFAWAZ et al., 2020; PEREZ-SANCHEZ et al, 2020).

Diante do exposto, o presente estudo tem por finalidade realizar uma análise da composição nutricional de produtos considerados nutricosméticos indicados para o tratamento dos cabelos.

\section{METODOLOGIA}

Foi realizada uma pesquisa exploratória e descritiva de nutricosméticos registrados e comercializados contra queda e fortalecimento dos cabelos em farmácias e lojas especializadas do setor de produtos saudáveis. Com relação aos critérios de inclusão, foram considerados: os produtos denominados para cabelos e unhas, apenas para o cabelo ou aqueles que além de beneficiar o cabelo e as unhas também apresentavam indicação para saúde da pele, que se apresentassem na forma de administração oral, seja comprimido, cápsula, pó ou gomas. Como critério de exclusão foram considerados os produtos que não apresentavam a composição completa no rótulo e os que são considerados medicamentos.

A coleta de dados foi realizada em farmácias alopáticas, drogarias e lojas de produtos saudáveis no município de Aracaju/SE, bem como em lojas virtuais nacionais de farmácias alopáticas, produtos naturais além de lojas de marcas específicas do setor reconhecidas no mercado. Para realização da coleta, foi elaborado um formulário para registro das informações acerca da descrição dos produtos, a qual baseou-se nos dados apresentados nos rótulos, a saber: nome comercial, marca, indicação de uso, posologia e composição nutricional com relação a composição nutricional. Os dados foram tabulados em planilhas e foram gerados gráficos no software Microsoft Excel. Para nível de análise, os dados da composição nutricional dos produtos foram agrupados em minerais, vitaminas e compostos bioativos.

\section{RESULTADOS}

Por meio da coleta foram obtidos 75 produtos, os quais com relação a função descrita no rótulo, $29 \%$ são específicos para o cabelo, 39\% para cabelos e unhas, e 32\% para cabelos, unhas e pele. Com relação a marcas dos produtos, foram encontradas 73 marcas diferentes, 22 delas correspondem aos produtos destinados ao cabelo, 27 referente a cabelo e unha, e 24 destinadas a cabelo, unha e pele. Ademais, nota-se que as posologias dos suplementos alimentares são semelhantes, com recomen- 
dações variando entre uma (52 produtos), duas (22 produtos) ou quatro (um produto) dosagens por dia. A composição nutricional e as proporções dos mesmos nutrientes eram similares em sua maioria.

As principais formas farmacêuticas encontradas foram de suplementos em cápsulas encontradas em 62 dos 75 produtos, seguido de comprimido (presente em dez produtos), goma (dois produtos) e em pó, apenas em um produto.

0 total de micronutrientes encontrados corresponde a 62, em que 13 deles foram minerais (Zinco, Cromo, Selênio, Silício, Iodo, Cobre, Magnésio, Manganês, Molibdênio, Fósforo, Ferro, Cálcio e Sódio), e 15 vitaminas (C, E, B6, B2, B1, A, B9, B7, D, B12, B3, B5, K, B8 e B9), além de 29 são bioativos (Extrato de Saw Palmetto, Extrato de Urtiga, Extrato de Ginkgo, Planta Esterois, Extrato de Gengibre, Taurina, Extrato de Ginseng Americano, Coenzima Q10, L-leucina, L-valina, Isoleucina, Ácido Hialurônico, Metilsufonilmetano, Ácido Linoleico, Cisteína, Cistina, Metionina, Nutricolin, Pantotenato de Cálcio, Actrisave, Ácido Ecosapentanoico, Ácido Docosahesanóico, Licopeno, Triptofano, Inositol, Colágeno, Luteína, Extracto de Cauda de Cavalo, Acido Oleico).

Diante da análise das informações adquiridas nos rótulos nutricionais dos produtos coletados, observou-se que alguns nutrientes se destacam com relação a sua presença na composição dos produtos, tais como a Vitamina B7 (Biotina) presente em 88\%, Vitamina B6 (Piridoxina) em 82\%, Zinco em $80 \%$, Selênio em 61\%, Vitamina C (Ácido Ascórbico) em 60\% e Vitamina E (Tocoferol) em $60 \%$ do total de produtos analisados, como mostra no Figuras 1 e 2.

Figura 1 - Principais micronutrientes encontrados e a relação do número de produtos que apresentam na sua composição

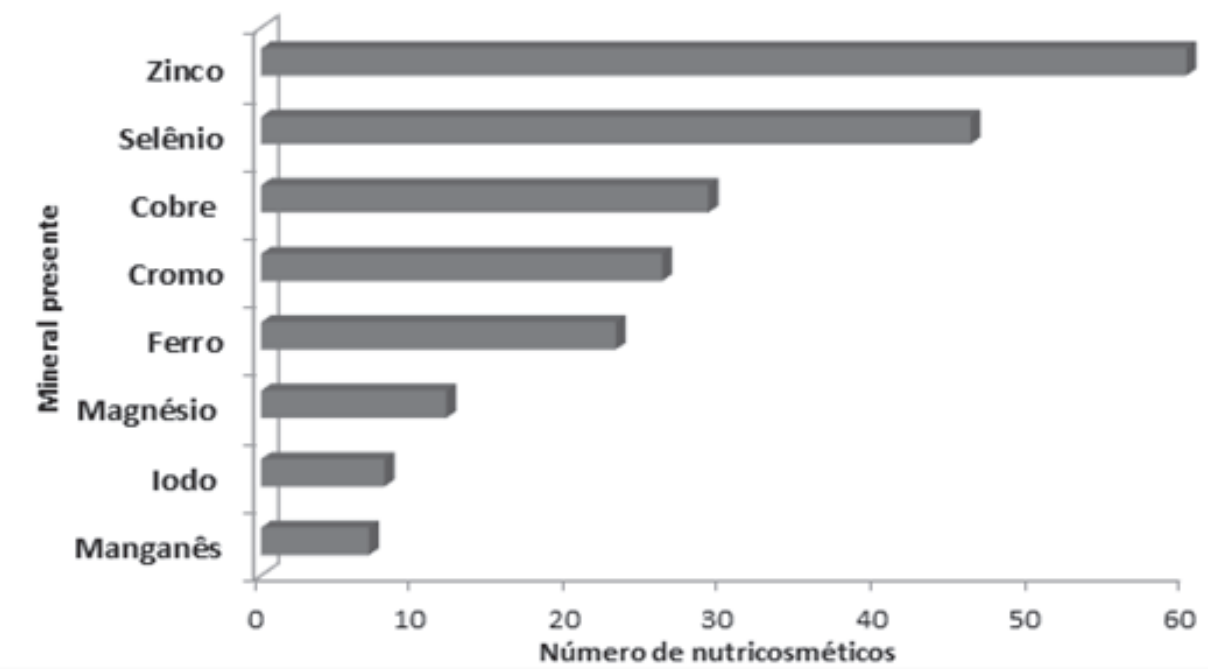

Fonte: Dados da pesquisa. 
Figura 2 - Principais vitaminas encontradas e a relação do número de produtos que apresentam na sua composição

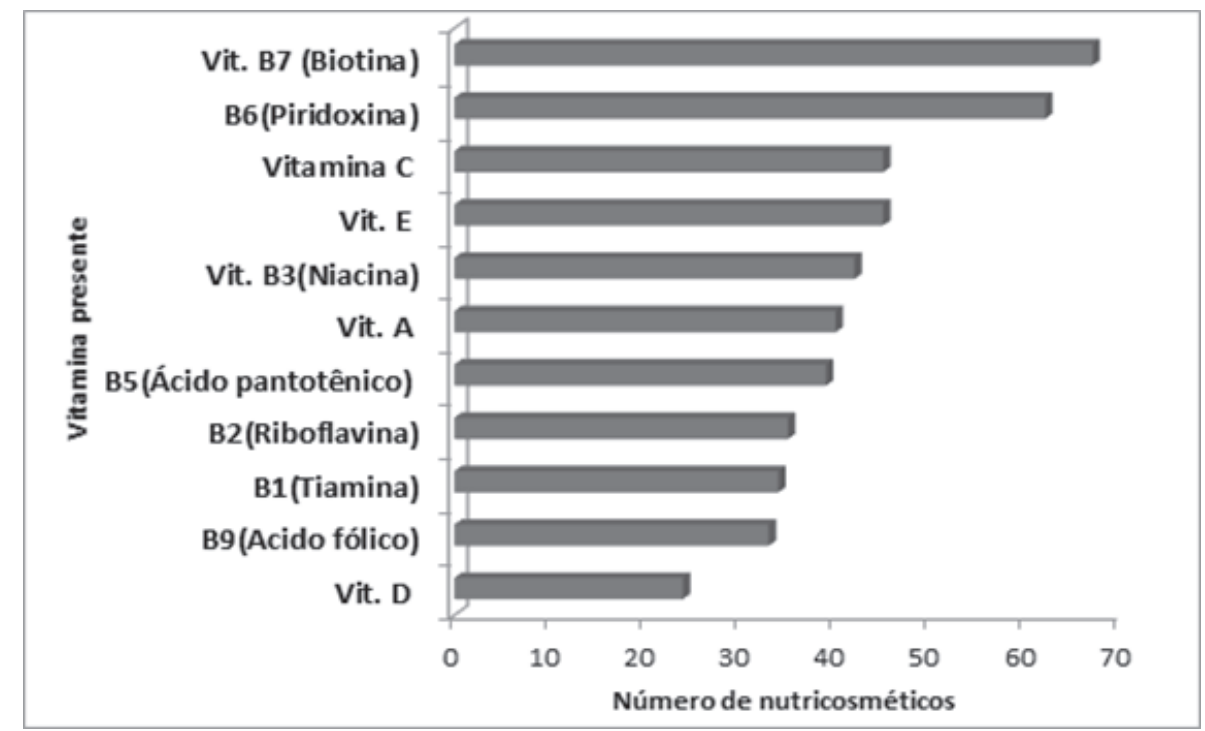

Fonte: Os Autores (2021).

Além dos minerais e vitaminas encontrados nos produtos, foram identificadas a presença de 29 compostos bioativos, no entanto, eles aparecem em poucos produtos. Os compostos que mais se destacaram foram: a Cisteína, presente em 7 nutricosméticos, a Metionina em 4, o Ácido Hialurônico em 3, a coenzima Q10 em 2, e os demais bioativos se encontram em 1 único produto.

\section{DISCUSSÃO}

Em virtude da escassez de estudos que avaliassem esse tipo de suplemento alimentar, serão descritas as funções biológicas dos principais nutrientes encontrados nos produtos analisados, bem como a relação com o tratamento de cabelo com base em evidências científicas.

Nesse sentido, destacam-se as vitaminas e os minerais, que além de desempenhar diversas funções fisiológicas fundamentais para o bom funcionando do organismo, também ajudam na saúde capilar (CRUZ et al., 2020; MATIAS, 2015).

\subsection{VITAMINAS DO COMPLEXO B}

As vitaminas do complexo B são imprescindíveis no desenvolvimento do cabelo, como as vitaminas B3 (Niacina), B6 (Piridoxina) e B7 (Biotina) que auxiliam na terapêutica da alopecia, sendo ingeri- 
das ou aplicadas dentro da pele. A biotina ajuda no tratamento da alopecia, sendo utilizada de forma isolada ou em conjunto com outros medicamentos. Além disso, ela proporciona firmeza no cabelo e a sua deficiência tem relação com problemas de queda dos fios, como a alopecia (HOSKING et al., 2019; FILETO et al., 2021; OSTROWSKA et al., 2019).

Uma pesquisa foi feita por Addor e colaboradores (2018) com mulheres que apresentavam Eflúvio Telógeno (ET), por um período de 180 dias, comparando a eficácia de dois produtos de uso oral, contendo alguns nutrientes, como as vitaminas do complexo B, para auxiliar na melhora do quadro. $O$ resultado mostrou que a ingestão de nutrientes adequados ajuda no tratamento do ET a partir do terceiro trimestre de uso. Ademais, o primeiro suplemento, contendo vitaminas do complexo $\mathrm{B}$, teve um melhor resultado em comparação com o outro produto que não continha essas vitaminas.

Outros estudos mostram a relação da deficiência da vitamina B7 com a queda capilar. Uma pesquisa elaborada por Trueb (2016), com 541 pacientes do sexo feminino, mostrou que 38\% dessas mulheres que apresentavam queda de cabelo possuíam deficiência dessa vitamina.

No estudo elaborado por Patel e colaboradores (2017), por sua vez, foram analisados 18 casos sobre o uso da suplementação de biotina para cabelo e unha. Em todos eles, foi relatada a existência de alguma patologia subjacente, como a alopecia, cuja presença foi constatada na maioria dos casos. Após alguns meses da suplementação do referido nutriente, foi possível perceber uma melhora no quadro dos participantes da pesquisa.

No entanto, não foram encontradas pesquisas suficientes que comprovem a eficácia da suplementação da biotina contra a queda capilar e em pessoas sem deficiência dessa vitamina. Apesar disso, é possível verificar o quanto esse nutriente está presente nos suplementos para a melhora dos cabelos, em que dos 75 produtos analisados, $88 \%$ deles continham essa vitamina (GUO; KATTA, 2017; MESINKOVSKA; BERGFED, 2013; SETTY et al., 2020).

A piridoxina desempenha diversas funções no organismo, duas delas são a participação como coenzima para a síntese de aminoácidos e a atuação na produção das hemácias e das células do sistema imunológico. A falta desse nutriente acarreta o aparecimento de anemia, distúrbios nervosos e problemas de pele. Sabe-se que a anemia pode afetar na saúde do cabelo levando a uma queda capilar (CARDOSO FILHO et al., 2019; DUGAN et al., 2021; HEMMINGER; WILLS, 2020). Devido a sua ação no organismo, nota-se o motivo dessa vitamina estar presente em $82 \%$ dos suplementos encontrados.

Foi feito um estudo com o uso oral da suplementação com extratos, minerais e as vitaminas do complexo B (B7, B1, B2, B3, B5, B6 e B12 - cobalamina) para o tratamento da alopecia androgenética feminina. A pesquisa durou 180 dias, e foi observado que $80 \%$ dos participantes notaram uma maior densidade e fortalecimento capilar (GRAU et al., 2015).

Em outro estudo realizado com 60 mulheres que tinham Eflúvio Telógeno, foi testada uma suplementação, contendo minerais e vitaminas do complexo B (B12, B1, B6, B2, B5, B7, entre outras), por um período de 180 dias, e houve uma regressão positiva do quadro (ADDOR et al., 2018). Importante ressaltar que a maioria dos estudos sobre o efeito da piridoxina no cabelo é sempre relacionado em combinação com outras vitaminas.

A literatura mostra que os casos encontrados referentes ao excesso de vitamina B6 acontecem devido ao uso de suplementos, não apenas a partir do consumo alimentar. A Piridoxina pode causar 
toxicidade se ultrapassar o valor de $1000 \mathrm{mg} / \mathrm{dia}$; no entanto, estudos já mostraram alguns sintomas a partir de doses menores que $500 \mathrm{mg} / \mathrm{dia}$. Os sintomas mais comuns são os neurológicos, como parestesia nas extremidades, ataxia, dormência e desequilíbrio (HEMMINGER; WILLS, 2020).

\subsection{VITAMINA C}

A vitamina $C$ é um antioxidante que auxilia no combate aos radicais livres, ajudando na proteção de moléculas como as proteínas e atua na formação do colágeno. Devido a sua atuação nos processos metabólicos de síntese proteica, o ácido ascórbico age de forma indireta no ciclo capilar. Apesar dos inúmeros benefícios, seu uso deve ser cauteloso, pois o seu excesso, acima de $2 \mathrm{~g} /$ dia, pode causar absorção excessiva de ferro, deficiência de vitamina B12 e distúrbios gastrointestinais. Por outro lado, a falta leva ao aparecimento de anemia, hemorragia e fragilidade capilar (ALVIM et al., 2014; SANTOS et al., 2019).

Não foram encontrados estudos que relacionam os níveis de vitamina $\mathrm{C}$ com a queda de cabelo, foram localizados apenas os que abordam a sua associação com a deficiência de ferro em pacientes com perda capilar (ALMOHANNA et al., 2019).

\subsection{VITAMINA E}

Também conhecida como tocoferol, é composta por tocoferóis e tocotrienóis e é extremamente importante para a saúde humana. Uma das suas funções é atuar como antioxidante, ajudando no combate aos radicais livres. A sua deficiência pode causar distúrbio neurológico progressivo, ataxia e deterioração muscular (BOCCARDI et al., 2016; FARINA et al., 2017; TRABER, 2014). 0 seu efeito no crescimento capilar ainda é pouco relevante (FINNER, 2013). Entretanto, $60 \%$ dos produtos encontrados apresentaram esse nutriente em sua composição.

Ademais, um estudo composto em sua maioria por homens, com a participação de 17 voluntários recebendo um medicamento placebo e outros 21 voluntários recebendo suplementação oral de $100 \mathrm{ml} /$ dia de tocotrienol (pertence à família da vitamina $\mathrm{E}$ ), por um período de 8 meses, concluiu que o grupo que fez 0 uso do suplemento teve uma melhora na quantidade de cabelo, comparado com o grupo placebo. Importante destacar que os voluntários apresentavam algum grau de perda capilar. Esse resultado positivo pode ter ocorrido devido ao poder antioxidante que esse composto tem, o qual ajudou na melhora do estresse sofrido pelo o couro cabeludo, que apresenta uma relação com alopecia (BEOY et al., 2010). Faz-se necessário mais estudos para elucidar o seu real efeito no crescimento capilar em pessoas saudáveis.

\subsection{ZINCO}

Por ser um íon intracelular que atua em associação com mais de 300 enzimas diferentes de várias classes, basicamente todo zinco está ligado a proteínas. Presente na carne de animais, em particular na carne vermelha e em aves, também é encontrado em alimentos à base de cereais, leites e derivados. Os sintomas adicionais de deficiência de zinco incluem alopecia e diversas formas de lesões cutâneas (MAHAN et al., 2013; WESSELLS et al., 2021). 
A maioria dos suplementos alimentares designados para o fortalecimento do cabelo e/ou unhas têm na sua composição zinco. Dentre os 75 produtos analisados, $80 \%$ deles apresentaram esse mineral, o qual normalmente se encontra associado a outros elementos, como o cobre ou vitaminas, como a biotina. Essa agregação possivelmente potencializa o resultado de fortalecimento, uma vez que cada um atua com um mecanismo diferente (MATIAS, 2015).

O seu uso frequente nos produtos é referente ao seu efeito na manutenção dos cabelos, pele e unhas, possivelmente devido a sua ação antioxidante e participação no metabolismo e síntese de proteínas, uma vez que o cabelo é composto em sua maioria por proteínas (GRASSI et al., 2021; MENÃO, 2020).

A literatura mostra o efeito benéfico do zinco em pacientes com alopecia areata. Um estudo feito por Ciampo e colaboradores (2018) com um menino de 11 meses que estava sofrendo com arcrodematite enteropática relata que o participante apresentou escassez no couro cabeludo. Também foi constatada uma deficiência de zinco, a partir disso, foi feita uma suplementação oral com esse mineral, de $2 \mathrm{mg} / \mathrm{kg} / \mathrm{dia}$, que resultou na reversão do quadro. Foi feita uma tentativa de retirada da suplementação e os sintomas reapareceram.

No entanto, são raros os estudos sobre o efeito da suplementação de zinco no crescimento capilar em pessoas que não possuem deficiência desse mineral. Importante destacar que o valor de referência é de $15 \mathrm{mg} /$ dia para adultos, e o excesso (valores maiores a $1 \mathrm{ou} 2 \mathrm{~g}$ ) pode causar sintomas, como vômitos, dores de cabeça e diarreia (AGNEW; SLESINGER, 2021; CRUZ et al., 2020; GUO; KATTA, 2017).

\subsection{SELÊNIO}

A insuficiência de selênio implica várias desordens no organismo, incluindo a alopecia, cabelos finos, hipopigmentação e riscas brandas nas unhas. Com a administração do selênio, é possível reverter estes sintomas, utilizando suplementos alimentares para a manutenção de cabelo e unhas normais, sua ação pode ocorrer devido ao seu efeito antioxidante e atuação com outros nutrientes, como as vitaminas C e E (CRUZ et al., 2020; MATIAS, 2015).

Apesar do selênio aparecer em $61 \%$ dos nutricosméticos pesquisados e ter ações importantes para a saúde do cabelo, pouco se sabe sobre a sua atuação no crescimento capilar em indivíduos que não apresentam deficiência desse mineral, sendo assim, é importante ter cautela, visto que o seu excesso pode causar prejuízos para a saúde (ALMOHANNA et al., 2019; MACFARQUHAR et al., 2010).

A recomendação diária é de 55 microgramas/dia para pessoas acima de 14 anos. 0 seu excesso (acima de 400 microgramas/dia) pode levar a uma toxicidade. Alguns dos sintomas são fraqueza, descoloração das unhas e queda capilar. Dessa forma, é fundamental o cuidado para que não ocorra uma ingestão excessiva desse mineral (ALMOHANNA et al., 2019).

\subsection{VITAMINA A}

A vitamina A auxilia na formação das unhas e dos cabelos, devido ao seu papel no estímulo da microcirculação cutânea (ROCHA et al., 2016). Apesar da sua deficiência levar a uma queda capilar, não há estudos comprovando a sua eficácia em casos de indivíduos com níveis normais dessa vitamina. 0 que se sabe é que o excesso pode levar a uma queda do cabelo (KATTA; HUANG, 2019). 
A recomendação diária dessa vitamina, segundo as Dietary Reference Intakes [Valores de Referência de Gestão de Nutrientes] (DRI), é de $900 \mathrm{mcg}$ (microgramas) para homens e $700 \mathrm{mcg}$ para mulheres, o valor tolerável é de $3000 \mathrm{mcg}$. No presente estudo, de 39 produtos que continham a vitamina A em sua composição, 2 indicavam em seus rótulos uma quantidade acima do valor tolerável (4000 e $5000 \mathrm{mcg}$ ) e um apresentava o valor exato de $3000 \mathrm{mcg}$. Esse dado é preocupante, uma vez que o excesso dessa vitamina pode causar perda capilar e unhas quebradiças (BURNS et al., 2020; PUJOL, 2011; RONIS et al, 2018; VAGULA; ROQUE, 2019).

\section{CONCLUSÃO}

Diante do exposto, pode-se compreender que existe um conjunto de micronutrientes como: As vitaminas do complexo $B$ (B3, B6 e B7), vitamina $C$, zinco, vitamina E, selênio, e vitamina $A$, que estão relacionados ao crescimento e fortalecimento dos cabelos. No entanto, vale destacar que os valores diários desses micronutrientes podem ser adquiridos por meio de uma alimentação balanceada, equilibrada e harmônica além de ser mais acessível, de forma que a suplementação deve ser orientada em casos de carências nutricionais.

Percebe-se que são necessários mais estudos que elucidem a relação dos nutricosméticos com o crescimento, fortalecimento e queda dos cabelos, visto que os relatos científicos encontrados não apresentam resultados conclusivos em indivíduos com bom aporte de nutrientes, mas, que há evidências em casos de patologias, como a calvície, entre outras.

Dos 75 produtos analisados, dois deles ultrapassaram o valor limite recomendado, dessa forma, altas dosagens de micronutrientes podem levar à toxicidade, trazendo prejuízos para a saúde do indivíduo, bem como promover queda capilar. Sendo assim, é imprescindível a orientação de um profissional na prescrição alimentar e nutricional para se evitar um consumo inadequado e o seu possível prejuízo a saúde.

\section{REFERÊNCIAS}

ADDOR, F.A.S.A. et al. Comparative evaluation between two nutritional Supplements in the improvement of telogen Effluvium. Clini Cosmet Invest Dermatol, n. 11, p. 431-436, 2018.

AGNEW, U. M.; SLESINGER, T. L. Zinc Toxicity. StatPearls, January, 2021.

ALFAWAZ, H. et al. Prevalence of dietary supplement use and associated factors among female college students in Saudi Arabia. BMC Womens Health, n. 1, v. 17, p. 1-7, 2017.

ALFAWAZ, H. et al. The prevalence and awareness concerning dietary supplement use among saudi adolescents. Int J Environ Res Pub He, n. 17, p. 1-14, 2020. 
ALMOHANNA, H. M. et al. The role of vitamins and minerals in hair loss: a review. Dermat Ther, v. 9, n. 1, p. 51-70, 2019.

ALVIM, S. A. F. et al. Influência da suplementação nutricional no tratamento do eflúvio telógeno: avaliação clínica e por fototricograma digital em 60 pacientes. Surg Cosmet Dermatol, v. 6, n. 2, p. 131-136, 2014.

BEOY, L. A. et al. Effects of tocotrienol supplementation on hair growth. Trop Life Sci Res, v. 21, n. 2, p. 91-99, 2010.

BOCCARDI, V. et al. Vitamin e family: role in the pathogenesis and treatment of Alzheimer's disease. Alzheimers Dement, n. 3, v. 2, p. 182-191, 2016.

BURNS, E. K. et al. Risks of Skin, Hair, and Nail Supplements. Dermatol Pract Concep, n. 4, v. 10, e2020089, p. 1-10, 2020.

CÂNDIDO, V. A. et al. Avaliação do perfil dos pacientes que frequentam os laboratórios de estética em relação ao uso de nutricosméticos. Saúde Meio Amb, v. 9, p. 97-105, 2020.

CARDOSO FILHO, O. et al. Vitaminas hidrossolúveis (B6, B12 E C): uma revisão bibliográfica. Rev Eletr Acervo Saúde, v. 11, n. 8, e285, p.1-7, 7 abr. 2019.

CIAMPO, I. R. L. et al. Acrodermatitis enteropathica: clinical manifestations and Pediatric diagnosis. Reva Paul Pediatr, n. 2, v.36, p. 238-241, 2018.

CRUZ, P. et al. Nutrição e saúde dos cabelos: uma revisão. Adv Nutrit Sci, v. 1, n. 1, p. 33-40, 2020.

DUGAN, C. et al. The misogyny of iron deficiency. Anaesthesia, n. 4, v. 76, 2021.

FARINA, N. et al. Vitamin e for alzheimer's dementia and mild cognitive impairment. Cochrane Db Syst Rev, v. 4, n. 4, p. 1-52, 2017.

FILETO, M. B. et al. Princípios ativos e procedimentos na Alopecia Androgenética, BWS Journal, v.4, p. 1-13, 2021.

FINNER, A. M. Nutrition and hair: deficiencies and supplements. Dermatol Clin, n. 1, v. 31, p. 16772, 2013.

GONÇALVES, M. S. L. Nutricosméticos e Cosmecêuticos: condicionantes regulamentares e posicionamento no mercado atual. 2016. 32f. Dissertação (Mestrado Integrado em Ciências Farmacêuticas) - Universidade de Coimbra, Coimbra. 2016. 
GRASSI, L. T. Espectroscopia de Raman e os efeitos do óleo em fibra capilar. Braz J Develop, v. 7, n. 5, p. 50478-50484, 2021.

GRAU, E. G. et al. Eficacia de un complemento alimenticio con serenoa serrulata y tocotrienoltocoferol frente a la alopecia androgenética y el efluvio telógeno femeninos: A propósito de un estudio piloto. Rev Arg Dermatol, v. 96, n. 1, p. 43-55, 2015.

GUO, E. L.; KATTA, R. Diet and hair loss: effects of nutrient deficiency and supplement use.

Dermatol Pract Concept, v. 1, n. 1, p.1-10, 2017.

HEMMINGER, A.; WILLS, B.K. Vitamin B6 Toxicity. Treasure Island (FL): StatPearls, April, 2020.

HOSKING, A. M. et al. Complementary and alternative treatments for alopecia: a comprehensive review. Skin Appendage Disord, v. 5, n. 2, p. 72-89, 2019.

KATTA, R.; RUANG, S. Supplements: na evidence-based approach. Skin Ther Letter, n. 5, v. 24, p.713, 2019.

MACFARQUHAR, J. K. et al. Acute selenium toxicity associated with a dietary supplement. Arch Internal Med, v. 170, n. 3, p. 2-11, 2010.

MAHAN, L. K. et al. Krause: alimentos, nutrição e dietoterapia. 13. ed. Rio de Janeiro: Elsevier, 2013.

MATIAS, B. A. T. Suplementos Alimentares para a Pele. 2015. 73f. Dissertação (Mestrado em Ciência Farmacêutica) - Instituto Superior em Ciências da Saúde, Egas Monis, Almada, Portugal. 2015.

MENÃO, T. F. Principais nutrientes de suplementos alimentares utilizados por idosos. 2020. $26 \mathrm{f}$. Trabalho de Conclusão de Curso (Graduação de Farmácia) -Universidade Cesumar - Maringá, 2020.

MESINKOVSKA, N. A.; BERGFELD, W. F. Hair: what is new in diagnosis and management? Female pattern hair loss update: diagnosis and treatment. Dermatol Clin, v. 31, n. 1, p.129-140, 2013.

MOTA, M. D. B. De Vênus a Kate Moss; reflexões sobre corpo, beleza e relações de gênero. Actas Diseño, v. 5, p. 100-104, 2008.

OSTROWSKA, M. et al. The effect of biotin interference on the results of blood Hormone assays. Endokrynol Polska, n.1, v. 70, p. 102-121, 2019.

PATEL, D. P. et al. A review of the use of biotin for hair loss. Skin Appendage Disord, v. 3, n.3, p. 166169, 2017. 
PEREZ-SANCHEZ et al. Safety concerns of skin, hair and nail supplements in retail stores. Cureus, $\mathrm{n}$. 7, v. 12, e9477, p. 1-11, 2020.

PUJOL, A. P. P. Nutrição aplicada à estética. Rio de Janeiro: Editora Rubio, 2011.

ROCHA, E. C. et al. A aplicação de alimentos antioxidantes na prevenção do envelhecimento cutâneo. Rev Cient FHO|UNIARARAS, v. 4, n. 1, p. 1-10, 2016.

RONIS, M. J. J. et al. Adverse effects of nutraceuticals and dietary supplements. Ann Rev Pharmacol Toxicol, v. 58, p. 1-22, 2018.

SANTOS, J. T. et al. Os efeitos da suplementação com vitamina C. Rev Conhec Online, v. 1, n. 11, p. 25, 2019.

SETTY, M. K. H. G. et al. Biotin interference in point of care HIV immunoassay. Biores v. 9, n. 1, p. 243-246, 2020.

TRABER, M. G. Vitamin e inadequacy in humans: causes and consequences. Adv Nutrit, v. 5, n. 5, p. 503-514, 2014.

TRUEB, R. M. Serum biotin levels in women complaining of hair loss. Int J Trichology, v.8, n. 2, p. 73-77, 2016.

VAGULA, J. M.; ROQUE, A. M. T. Alimentação e qualidade de vida. Londrina: Editora e Distribuidora Educacional S.A., 2019.

WESSELLS, K. R. et al. Plasma and nail zinc concentrations, but not hair zinc, respond positively to two different forms of preventive zinc supplementation in young laotian children: a randomized controlled trial. Biol Trace Elem Res, v. 199, n. 2, p. 442-452, 2021.

WITT, Z. G. S. J.; SCHNEIDER, P. A.; Nutrição Estética: valorização do corpo e da beleza através do cuidado nutricional. Ciên Saúde Col, v. 16, n. 9, p. 3909-3916, 2011. 
1 Graduanda em Nutrição. Universidade Tiradentes - UNIT, Aracaju, SE. Brasil. Email: nathaliamandarino@hotmail.com

2 Graduanda em Nutrição. Universidade Tiradentes - UNIT, Aracaju, SE. Brasil. Email: ingrid_gabriele18@hotmail.com

3 Nutricionista. Universidade Tiradentes - UNIT, Aracaju, SE. Brasil. Email: Eliane.freire.nutri@gmail.com

4 Doutor em Biotecnologia Industrial. Universidade Tiradentes - UNIT, Aracaju, SE. Brasil. ORCID 0000-0002-92341085. Email: heribertoanjos@yahoo.com.br

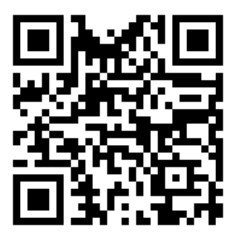

A autenticidade desse artigo pode ser conferida no site https://periodicos. set.edu.br

\section{(ㄷ) (1) (2)}

Este artigo é licenciado na modalidade acesso abertosob a Atribuição-Compartilhalgual CC BY-SA

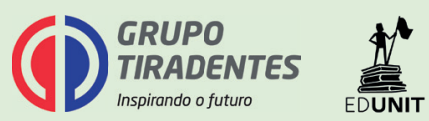

